\title{
Sargan's Instrumental Variable Estimation and GMM
}

\author{
Manuel Arellano \\ CEMFI \\ Working Paper No. 0110 \\ December 2001
}

This article is dedicated to the memory of Denis Sargan. I am grateful to Olympia Bover, Eric Ghysels, Alastair Hall, David Hendry, Peter Phillips, and Enrique Sentana for helpful comments and discussions. (Email address: arellano@cemfi.es).

CEMFI, Casado del Alisal 5, 28014 Madrid, Spain.

www.cemfi.es 


\begin{abstract}
This paper surveys J. D. Sargan's work on instrumental variable estimation and its connections with the generalized method of moments. I first present the modelling context in which Sargan motivated instrumental variable estimation. Then I review the theory of instrumental variable estimation as developed in Sargan (1958). I discuss his approach to efficiency, his minimax estimator, tests of over- and under-identification, and his later work on the finite sample properties of IV estimators. Next, Sargan's approach to modelling IV equations with serial correlation is discussed and compared to the GMM approach. Finally I describe Sargan's (1959) results for non-linear in parameters IV models.
\end{abstract}

Keywords: Minimax estimation; Finite sample properties; Error in variables; Serial correlation; Non-linear models.

JEL Classification: C10, C12, C13. 
"I pretty early realized that the Geary method was very close to LIML except he was using arbitrary functions of time as the instrumental variables, (...). One could easily generalize the idea to the case, for example, of using lagged endogenous variables to generate the instrumental variables. That is really where my instrumental variable estimation started from. I was actually using it to estimate macroeconomic models fairly early, but the models didn't turn out very interesting to my way of thinking. I developed various ideas on time lags at that stage, and I actually had an early version of the Phillips curve in my model. I spent a lot of those years when I had spare time using an electric Marchand calculating machine in a Leeds University basement and getting out estimates which I didn't get published myself."

(J. D. Sargan, interviewed by P. C. B. Phillips, 1985.)

\section{Introduction}

This paper surveys Sargan's work on instrumental variable estimation and its connections with the generalized method of moments. Denis Sargan pursued his interests in instrumental variable estimation all through his career. He focused in particular on asymptotic expansions of the distributions of estimators and test statistics. However, his two articles of 1958 and 1959 provided a fully developed theory of instrumental variable estimation which directly connects with the GMM perspective of the 1980s. So it seems natural to concentrate on those articles for the purpose of this survey [see Maasoumi (1988) for an account of Sargan's contributions to econometrics].

There is a modelling connection between GMM and Sargan's focus on moment conditions that do not necessarily provide a complete description of the probability distribution under consideration. There is also a statistical connection since Hansen's (1982) treatment of GMM estimation built on Sargan's results. Specifically, Hansen's GMM class of estimators generalized Sargan's class of linear and non-linear IV estimators, and Hansen's analysis of efficiency followed Sargan's approach based on an optimal selection matrix for the moment conditions.

There is, however, an important aspect of Hansen's GMM approach, other than greater generality, that was not present in Sargan's work. Hansen proposed to use GMM estimators based on moment conditions that exhibited dependence over time, constructed to be robust to the unmodelled components. To that 
end he suggested to use a weighting matrix that took account of the temporal dependence. This suggestion had both modelling and statistical implications. I examine the modelling implications below in connection with Sargan's work on instrumental-variable models with serial correlation. A statistical implication was that consistent estimators of the covariance matrix of the sample moment conditions were required. Consistent estimators under various forms of time series dependence were suggested in Hansen (1982) and other papers in the literature.

In the early 1950s error in variables problems and simultaneous equations were pursued in two different literatures. The instrumental variable technique was associated with an ad-hoc cure for measurement error. LIML had been developed for estimation of a single structural equation of a simultaneous system by Anderson and Rubin $(1949,1950)$, the mathematical analogy between the IV and LIML methods had been first noted by Durbin (1954), and 2SLS was being introduced by Basmann (1957) and Theil (1961). In fact, it was also being introduced by Sargan (1958) as an instrumental variable estimator in a more general context. Sargan was not only providing a definitive analysis of the instrumental variable method: He was also using it to put together simultaneity and error in variables, he was providing an IV analogue of LIML as a minimax estimator, and in his 1959 article he was developing nonlinear-in-parameters IV estimation and its properties.

Sargan was thinking in terms of moment conditions, overidentifying restrictions, and partially specified models. He was also considering issues of choice of instruments, finite sample biases, and underidentification. Many of the themes that appeared with renewed impetus in the econometrics literature of the 1980's and 1990's were present in a surprisingly mature way in Sargan's 1958 and 1959 articles. And yet, relatively little attention was paid to this way of thinking about econometric estimation for the next 20 years. Most of the textbooks of the 1960's routinely surveyed simultaneous equations estimators (2SLS, 3SLS, LIML, FIML) as distinct from instrumental variable methods, which were only alluded to in a casual way in the context of discussions of measurement error (although Hausman 1975 and Hendry 1976 both linked IV to FIML in each direction). Moreover, when in the mid 1970's the literature on nonlinear structural models began to develop, the connection with Sargan's (1959) article was often overlooked. 
The paper is organized as follows. Section 2 presents the modelling context in which Denis Sargan motivated instrumental variable estimation. Section 3 reviews the theory of instrumental variable estimation and inference as developed by Sargan (1958). I discuss his approach to efficiency, his minimax estimator, tests of over- and under-identification, and his later work on the finite sample properties of IV estimators. In Section 4 Sargan's approach to modelling IV equations with serial correlation is discussed and compared to the GMM approach. In Section 5 I describe Sargan's (1959) results for non-linear in parameters IV models. Finally, Section 6 contains some concluding remarks.

\section{Models and Instruments}

The Model Sargan (1958) considered a structural equation of the form

$$
\alpha_{0}^{\prime} w_{t}^{*}=\varepsilon_{t}
$$

where the vector $w_{t}^{*}$ (of order $q+1$ ) contained both endogenous and predetermined variables, and $\varepsilon_{t}$ was a structural random shock that was assumed to be independent of all the predetermined variables in the complete system.

The variables were observed with error:

$$
w_{t}=w_{t}^{*}+v_{t}
$$

where $v_{t}$ is a vector of measurement errors. In the case of variables measured without error the corresponding elements of $v_{t}$ are zero. Sargan interpreted the variables $w_{t}^{*}$ as the "actual variables to which the economic agents react". Thus, he argued that it was not necessarily true that "the determined variable is also an ideal economic variable in the sense that it is exactly equal to the variable to which some other economic agent later reacts, or that if an economic variable appears as a cause in two different equations the appropriate values of the ideal economic variable are the same" (1958, p. 395).

Combining the two equations above a relationship among the observed time series was obtained

$$
\alpha_{0}^{\prime} w_{t}=u_{t}
$$


in which the equation's error $u_{t}$ contains both a structural shock and measurement errors:

$$
u_{t}=\varepsilon_{t}+\alpha_{0}^{\prime} v_{t}
$$

The Instruments Sargan assumed the availability of some predetermined variables (a vector of instrumental variables of order $r$ denoted $z_{t}$ here) whose measurement errors were independent of $v_{t}$ and $\varepsilon_{t}$. This requirement excluded: (1) the predetermined variables in the relationship (unless measured without error), (2) lags of variables in the equation ("unless -according to Sargan- one makes the unrealistic assumption that measurement errors are not autocorrelated"), and (3) any predetermined variable which is constructed from the same data as one of the variables in the equation. Sargan concluded that it was necessary that the sources of data used for constructing the instrumental variables should be largely independent of those used to construct the variables in the equation.

Thus the only variables in the equation that could be used as instrumental variables were predetermined variables with zero measurement errors, like the constant term, trends, and seasonal components.

He then suggested to follow Reiers $\varnothing \mathrm{l}$ (1945) and base estimation on the sample orthogonality conditions

$$
\frac{1}{T} \sum_{t=1}^{T} z_{t} u_{t} \equiv\left(\frac{1}{T} \sum_{t=1}^{T} z_{t} w_{t}^{\prime}\right) \alpha=0,
$$

which provided $r$ equations for the $q$ ratios of the coefficients, so that if $r=q$ they give a unique set of estimates of the coefficients $\alpha_{0}$.

Sargan expressed concern about the real possibilities of this set of assumptions for identifying parameters of interest. He wrote: "It is not easy to justify the basic assumption concerning these errors, namely, that they are independent of the instrumental variables. It seems likely that they will vary with a trend and with a trade cycle. In so far as this is true the method discussed here will lead to biased estimates of the coefficients. Nothing can be done about this since presumably, if anything were known about this type of error, better estimates of the variables could be produced" (1958, p. 396). 
From the macro data at his disposal and following Stone (1947), Sargan argued that there were effectively no more than three factors that could be used as instrumental variables: a linear trend, the ten year business cycle, and the rate of change of the ten year business cycle. If there were large random events (of the same order of magnitude as the cyclical movements) such as strikes and wars, and if the structural errors could be regarded as independent of these events, then this might allow the identification of further coefficients, but he felt this possibility was "very rarely realistic". And he concluded: "In practice, when data covering less than 20 years are used, it seems appropriate to use three instrumental variables: a linear trend, a lagged variable that leads in the trade cycle, and a lagged variable that lags with reference to the trade cycle. Analyses of single economic time series indicate that if longer periods of time were studied a factor analysis might disclose more general factors".

At the time, Sargan was particularly interested in wage-price inflation models. He was skeptical about the existence of a stable trade-off between inflation and unemployment (see Desai, Hendry and Mizon 1997). In modelling wages, Sargan emphasized the role of union behavior and real wage resistance in wage bargaining: "I couldn't quite believe in the Phillips curve and some of the conclusions that were drawn about the ease with which a small increase in unemployment would cure inflation" (P. C. B. Phillips 1985, ET Interview). Reading his 1964 Colston paper, one senses that Sargan, who devoted much time to estimating macro models in those years, was probably left with the feeling that the instrumental variable method delivered less than he had initially hoped.

Errors in the Variables versus Errors in the Equations The basic motivation for the instrumental variable approach adopted by Sargan (1958) was to deal with equations that exhibited both simultaneity and measurement errors in exogenous (or predetermined) variables.

In contrast with early econometric practice, the Cowles commission approach to econometrics stressed errors in the equations and simultaneity biases as opposed to errors in variables and measurement error biases. This switch of emphasis took place even though the relative importance of simultaneity and measurement error 
had not been clearly established in many empirical areas (see Goldberger 1972, p. 993, and Heckman 2000, pp. 71-72, for discussions of these issues). In this respect, by giving symmetric consideration to the two types of errors, Sargan's (1958) paper was an exception.

A particularly attractive feature of Sargan's instrumental-variable framework -that has become common place in modern econometric practice- was to make operational the notion that measurement error in predetermined variables need not result in lack of identification, provided that the structural model contains sufficient overidentifying restrictions (Golberger 1972, p. 996).

Linearity in variables is an essential ingredient to the possibility of adding up errors in variables and errors in equations. Extending his 1958 results, Sargan (1959) considered IV models that were non-linear in parameters but linear in variables. Beginning with the work of Amemiya (1974) and Jorgenson and Laffont (1974), Hansen (1982) and a sizeable part of the GMM literature emphasized fully non-linear relationships, but in this process the measurement-error perspective was lost.

\section{The IV Estimation Method}

Early contributions to the instrumental-variable method were made by Wald (1940), Reiersøl (1945), and Geary (1948,1949). Before them, the IV method can also be associated with the work of Working (1927), P. G. Wright (1928), and S. Wright (1934) [see Goldberger (1972) for a survey of the work of Sewall Wright]. The papers by Chernoff and Rubin (1953) and Durbin (1954) made contributions that were more closely related to subsequent work by Sargan. These papers were concerned with the connection between simultaneous equations and measurement error. Durbin (1954) discussed alternative processes generating measurement errors and pointed out the analogy between the overidentified IV case and Anderson and Rubin's method for a single structural equation. Chernoff and Rubin's chapter in Cowles Monograph 14 suggested a modification of LIML through which predetermined variables with measurement error were treated as endogenous variables. 
The contributions of Sargan's (1958) paper were to provide a definitive treatment of the IV method and to establish its asymptotic properties. A review of these contributions is given in this section. Sargan (1958), like Hansen (1982), considered the asymptotic properties of a class of econometric estimators that are defined in terms of orthogonality conditions. This was a bold partial-information approach that focused estimation on objects of economic interest, abstracting from other features of the probability distribution of the variables under consideration. It also made it possible to define optimality in a well defined sense, even if the resulting optimal estimators were not necessarily asymptotically efficient by comparison with full information methods.

\subsection{Asymptotic Properties}

Sargan (1958) assumed that the $r \times 1$ vector of sample orthogonality conditions

$$
g_{T}=\frac{1}{\sqrt{T}} \sum_{t=1}^{T} z_{t} u_{t}
$$

had an asymptotic joint normal distribution of the form

$$
g_{T} \stackrel{d}{\rightarrow} \mathcal{N}\left(0, \sigma^{2} \bar{M}_{z z}\right)
$$

where $\sigma^{2}=E\left(u_{t}^{2}\right)$ and $\bar{M}_{z z}$ denotes the stationary limit of $E\left(z_{t} z_{t}^{\prime}\right)$ given by

$$
\bar{M}_{z z}=\lim _{T \rightarrow \infty} E\left(\frac{1}{T} \sum_{t=1}^{T} z_{t} z_{t}^{\prime}\right)=p \lim _{T \rightarrow \infty} \frac{1}{T} \sum_{t=1}^{T} z_{t} z_{t}^{\prime} .
$$

This was based on the assumption that the variables were stationary and that $u_{t}$ was independent of $z_{s}$ for $t \geq s$ and of $u_{s}$ for $t \neq s$, for in this case:

$$
E\left(g_{T} g_{T}^{\prime}\right)=\frac{1}{T} \sum_{t=1}^{T} \sum_{s=1}^{T} E\left(u_{t} u_{s} z_{t} z_{s}^{\prime}\right)=\sigma^{2} E\left(\frac{1}{T} \sum_{t=1}^{T} z_{t} z_{t}^{\prime}\right)
$$

Let us introduce some normalization so that $u_{t}=w_{t}^{\prime} \alpha_{0}=y_{t}-x_{t}^{\prime} \beta_{0}$ where $\beta_{0}$ is a $q \times 1$ vector of parameters, and the usual matrix notation $Z^{\prime} X=\sum_{t=1}^{T} z_{t} x_{t}^{\prime}$, etc. Moreover, $\bar{M}_{z x}$ and $\bar{M}_{z w}$ denote the probability limits of $Z^{\prime} X / T$ and $Z^{\prime} W / T$, 
respectively. In the just-identified case, that is, when $r=q$ and $\bar{M}_{z x}$ has full rank, Sargan (1958) showed that the simple IV estimator

$$
\widehat{\beta}=\left(Z^{\prime} X\right)^{-1} Z^{\prime} y
$$

has limiting distribution

$$
\sqrt{T}\left(\widehat{\beta}-\beta_{0}\right) \stackrel{d}{\rightarrow} \mathcal{N}\left(0, \sigma^{2} \bar{M}_{z x}^{-1} \bar{M}_{z z} \bar{M}_{x z}^{-1}\right) .
$$

When there are more instruments than unknown parameters, that is, when $r>q$, he considered $q$ linear combinations of the instruments

$$
z_{t}^{*}=\Theta z_{t}
$$

where $\Theta$ is a $q \times r$ matrix of coefficients. Now from the previous discussion the asymptotic variance of the IV estimator based on $z_{t}^{*}$ is

$$
\sigma^{2}\left(\Theta \bar{M}_{z x}\right)^{-1}\left(\Theta \bar{M}_{z z} \Theta^{\prime}\right)\left(\bar{M}_{x z} \Theta^{\prime}\right)^{-1} .
$$

Then Sargan showed that the optimal choice for $\Theta$ that minimizes the asymptotic variance can be taken to be

$$
\Theta=\bar{M}_{x z} \bar{M}_{z z}^{-1}
$$

in which case we get

$$
\sigma^{2}\left(\bar{M}_{x z} \bar{M}_{z z}^{-1} \bar{M}_{z x}\right)^{-1} .
$$

Since $\Theta$ is unknown, he argued, the previous result suggests consideration of $\widehat{\Theta}=X^{\prime} Z\left(Z^{\prime} Z\right)^{-1}$ giving rise to the estimator that solves

$$
\left(X^{\prime} Z\left(Z^{\prime} Z\right)^{-1} Z^{\prime} X\right) \widehat{\beta}=X^{\prime} Z\left(Z^{\prime} Z\right)^{-1} Z^{\prime} y
$$

which has the optimal asymptotic variance. Sargan suggested to use the estimated variance matrix

$$
\widehat{\sigma}^{2} T\left(X^{\prime} Z\left(Z^{\prime} Z\right)^{-1} Z^{\prime} X\right)^{-1}
$$

where $\widehat{\sigma}^{2}=\widehat{u}^{\prime} \widehat{u} / T$ and $\widehat{u}=y-X \widehat{\beta}$.

Sargan also noted that for each normalization a different set of estimates is obtained. He showed that these estimates differ asymptotically by quantities of order $1 / T$ provided that $\bar{M}_{w z} \bar{M}_{z z}^{-1} \bar{M}_{z w}$ is of rank $q$. The estimator (3.7) is, of course, the two-stage least squares estimator, which at the time was being independently introduced from a different perspective by Basmann and Theil. 


\subsection{Sargan's Minimax IV Estimator}

The motivation for the minimax method was to obtain a symmetrically normalized instrumental variable estimator for the overidentified case, after noting the lack of invariance to normalization of estimates of the form (3.7) that were obtained by deleting an arbitrary equation from the set of optimal sample moment conditions.

Since the model posits zero correlation between errors and instruments, the idea was to choose as estimator of $\beta_{0}$ the value that minimizes the maximum possible sample correlation between the errors and a linear combination of the instruments.

Let us consider arbitrary linear combinations of the instruments

$$
m_{t}(\gamma)=z_{t}^{\prime} \gamma
$$

and of the variables appearing in the econometric model

$$
u_{t}(\alpha)=w_{t}^{\prime} \alpha
$$

The squared sample correlation between $m_{t}(\gamma)$ and $u_{t}(\alpha)$ is given by

$$
\rho^{2}(\alpha, \gamma)=\frac{\left(\alpha^{\prime} W^{\prime} Z \gamma\right)^{2}}{\left(\alpha^{\prime} W^{\prime} W \alpha\right)\left(\gamma^{\prime} Z^{\prime} Z \gamma\right)}
$$

For given $\alpha$, the maximum correlation is given by

$$
\lambda(\alpha)=\max _{\gamma} \rho^{2}(\alpha, \gamma)=\frac{\alpha^{\prime} W^{\prime} Z\left(Z^{\prime} Z\right)^{-1} Z^{\prime} W \alpha}{\alpha^{\prime} W^{\prime} W \alpha} .
$$

Thus, the resulting estimator is given by solving the minimax problem

$$
\min _{\alpha}\left[\max _{\gamma} \rho^{2}(\alpha, \gamma)\right]=\min _{\alpha} \lambda(\alpha)=\widehat{\lambda}_{1}
$$

subject to a normalization restriction. The statistic $\hat{\lambda}_{1}$ is the smallest eigenvalue of the matrix $W^{\prime} Z\left(Z^{\prime} Z\right)^{-1} Z^{\prime} W$ in the metric of $W^{\prime} W$.

The minimax estimator provided an instrumental-variable analogue and a generalization of Anderson and Rubin's limited-information maximum likelihood 
method (LIML). Sargan (1958, Sec. 13) noted that "the LIML method is equivalent to using the instrumental variables method with all the predetermined variables in the model used as instrumental variables. This procedure is reasonable since an essential assumption of the LIML method is that there are no measurement errors."

Thus, Sargan's conclusion was that in the context of a structural equation subject to measurement error one can still use symmetrically normalized IV estimators but these lack the LIML interpretation, and the validity of instruments depends on the measurement error properties of the model, so that it is no longer true that necessarily all the predetermined variables in the system are valid instruments.

Sargan's minimax estimator can also be regarded as minimizing the largest standardized linear combination of the sample moments:

$$
\min _{\alpha}\left[\max _{\gamma} \frac{\left(u(\alpha)^{\prime} Z \gamma\right)^{2}}{\gamma^{\prime} V(\alpha) \gamma}\right]=\min _{\alpha}\left[u(\alpha)^{\prime} Z\right] V(\alpha)^{-1}\left[Z^{\prime} u(\alpha)\right]
$$

where $V(\alpha)=\operatorname{Var}\left[Z^{\prime} u(\alpha)\right]$ and the previous equality follows from application of the Cauchy-Schwarz inequality. For $V(\alpha)=u(\alpha)^{\prime} u(\alpha)\left(Z^{\prime} Z\right)$ we obtain Sargan's estimator, but for choices of $V(\alpha)$ based on alternative assumptions about the form of the variance matrix of the moments we may obtain other generalized minimax or "continuously updated" GMM estimators of the type considered by Hansen, Heaton, and Yaron (1996).

\subsection{Inference}

Testing Over-identifying Restrictions Sargan (1958) proposed a specification test of the existence of a relationship that satisfied all moment conditions. He showed that as long as $\bar{M}_{w z}$ has reduced rank $q$ :

$$
T \widehat{\lambda}_{1} \stackrel{d}{\rightarrow} \chi_{r-q}^{2}
$$

This provided an instrumental-variables analogue to one of the criteria derived by Anderson and Rubin $(1949,1950)$ for testing over-identification in a single equation from a system of simultaneous equations. 
As a sketch of the argument, note that factoring $\left(Z^{\prime} Z / T\right)^{-1}=C C^{\prime}$, in view of (3.1) and the consistency of $\widehat{\sigma}^{2}, C^{\prime} g_{T} / \widehat{\sigma} \stackrel{d}{\rightarrow} N\left(0, I_{r}\right)$. Moreover, letting $G=$ $C^{\prime}\left(Z^{\prime} X / T\right)$ we have

$$
h=\frac{T^{-1 / 2} C^{\prime} Z^{\prime} \widehat{u}}{\widehat{\sigma}}=\left[I_{r}-G\left(G^{\prime} G\right)^{-1} G^{\prime}\right] \frac{C^{\prime} g_{T}}{\widehat{\sigma}} .
$$

Since the limit of $\left[I_{r}-G\left(G^{\prime} G\right)^{-1} G^{\prime}\right]$ is idempotent and has rank $r-q$, it follows that

$$
h^{\prime} h=T \frac{\widehat{u}^{\prime} Z\left(Z^{\prime} Z\right)^{-1} Z^{\prime} \widehat{u}}{\widehat{u}^{\prime} \widehat{u}} \stackrel{d}{\rightarrow} \chi_{r-q}^{2},
$$

and hence also (3.13), since due to their asymptotic equivalence the same result holds if $\widehat{u}$ is replaced by the minimax residual.

A statistic like $T \hat{\lambda}_{1}$ or $h^{\prime} h$ has become known as a "Sargan test" and it is a standard complement when reporting IV estimates. Sargan argued "this provides a significance test for the hypothesis that there is a relationship between the suggested variables with a residual independent of all the instrumental variables"; and he added, "this is a suitable test even when $\bar{M}_{w z}$ is of rank less than $q$ since it can be shown that in this case the probability of rejecting the hypothesis will be less than in the other case".

Sargan (1959) considered a generalization to nonlinear-in-parameters IV models, and Hansen (1982) extended this type of specification test to a general nonlinear GMM environment with dependent observations (often called a " $J$ test").

Testing for Underidentification Next, Sargan considered a test of underidentification, that is, a test of the hypothesis of the existence of a multiplicity of relationships that satisfy all moment conditions. This is a test of the null that the rank of $\bar{M}_{w z}$ is less than $q$ against the alternative that the rank is equal to $q$. This type of test was also considered by Koopmans and Hood (1953) in the context of a single-equation from a simultaneous system.

Koopmans and Hood, and Sargan stated that when $\bar{M}_{w z}$ has rank $q-1$, letting $\hat{\lambda}_{2}$ be the second smallest characteristic root, we have

$$
T\left(\hat{\lambda}_{1}+\hat{\lambda}_{2}\right) \stackrel{d}{\rightarrow} \chi_{2(r-q)+2}^{2} .
$$


Thus, this result could be used as a test of the hypothesis that the equation is underidentified and that any admissible equation has a homoskedastic and nonautocorrelated error. Sargan (1958) pointed out that "this hypothesis is not very likely to be true a priori since even if there is a relationship between the suggested variables with a non-autocorrelated residual it is unlikely that there would be a second combination of these variables not only independent of all instrumental variables but non-autocorrelated as well". He regarded, however, the use of the test as "a useful qualitative answer as to whether the estimates are reasonably well identified".

If $\bar{M}_{w z}$ has rank $q-1$, there is another solution $\alpha_{0}^{*}$ not proportional to $\alpha_{0}$ that satisfies the original moment equations:

$$
E\left(z_{t} w_{t}^{\prime}\right)\left(\alpha_{0}, \alpha_{0}^{*}\right)=0
$$

So, a test of underidentification can be regarded as a test of the overidentifying restrictions in (3.16) subject to an extended normalization of $\left(\alpha_{0}, \alpha_{0}^{*}\right)$. In fact, letting $A=\left(\alpha, \alpha^{*}\right)$, it turns out that $T\left(\widehat{\lambda}_{1}+\widehat{\lambda}_{2}\right)$ coincides with the minimizer of

$$
T\left(\alpha^{\prime} W^{\prime} Z, \alpha^{* \prime} W^{\prime} Z\right)\left(A^{\prime} W^{\prime} W A \otimes Z^{\prime} Z\right)^{-1}\left(\begin{array}{c}
Z^{\prime} W \alpha \\
Z^{\prime} W \alpha^{*}
\end{array}\right)
$$

subject to $A^{\prime} W^{\prime} W A=I_{2}$. This has been shown by Arellano, Hansen, and Sentana (1999), who used the idea to consider tests of underidentification from a GMM perspective in a wider context.

\subsection{Approximating the Distribution of IV Estimates}

Sargan (1958) stressed the distinction between the purely theoretical asymptotic results and the accuracy of the asymptotic approximations for finite $T$. He provided a discussion of the determinants of the quality of the asymptotic approximation and offered specific recommendations for practitioners. On the basis of unpublished calculations he asserted that the biases of IV estimates and $T \widehat{\lambda}_{1}$ were of order $r / T \lambda_{2}$ where $\lambda_{2}$ is the population counterpart of $\hat{\lambda}_{2}$. The implication was that the asymptotic approximation was poor when the relationship was almost 
unidentified, and when the number of instruments was large relative to the sample size.

Sargan discussed the issue of instrument choice pointing out a finite sample trade-off between bias and efficiency. He argued that although the addition of a new instrumental variable will not worsen the asymptotic variance matrix, "the improvements are usually small after the first three or four instrumental variables have been added. Thus there may be no great advantage in increasing the number of instrumental variables, and (...) it emerges that the estimates have large biases if the number of instrumental variables becomes too large" (p. 400). His practical suggestion was to require that $r \leq T / 20$. In a similar vein he also suggested a crude finite-sample adjustment to the chi-squared statistic. Finally, he compared IV with OLS, arguing that although theoretically IV is better than OLS because of consistency, for finite $T$ the advantage of IV is less certain since "the instrumental variable estimates may have large biases especially in the almost unidentified case and in the event the number of instrumental variables is large".

Improved Asymptotic Approximations The use of asymptotic expansions to obtain improved approximations to the distributions of econometric estimators was pioneered by Sargan. The first paper published in this area was Sargan and Mikhail (1971), which developed an Edgeworth (or Gram-Charlier) approximation to the distribution of instrumental variables estimates and evaluated the accuracy of the approximation for a model with two endogenous variables [the abstract of an earlier version of this paper was published in Econometrica in 1964]. In subsequent work, Sargan (1975a) approximated the distribution of $t$ ratios of IV estimators, and proved a general theorem on the validity of Edgeworth expansions for statistics that are defined as functions of a vector of more primitive statistics. The 1975 paper also contained an early discussion of empirical Edgeworth approximations. The results of these two papers relied on "classical assumptions" in the sense that excluded lagged endogenous variables and nonnormal errors. The general conclusions were that "the asymptotic approximation will be particularly poor if $r / T$ is not small, and if the variance of the reduced form errors is relatively large. In addition increased correlation between the re- 
duced form and equation errors worsen the asymptotic approximation" (Sargan 1975a, p. 340).

In an important paper, Sargan (1976) obtained explicit formulae for the secondorder Edgeworth expansion of a statistic that is defined as a smooth function of sample second moments of the data. These formulae covered models containing lagged dependent variables (a case which was also independently considered by Phillips 1977). To summarize the setting of Sargan's results, let $\phi(p)$ represent a scalar estimator or test statistic as a function of a vector $p$ of sample second moments that have been generated by some stationary stochastic process. If a CLT is available for $p: \sqrt{T}(p-\mu) \stackrel{d}{\rightarrow} \mathcal{N}(0, \Sigma)$, using the "delta method", a first-order approximation to the $c d f$ of $b_{T}=\sqrt{T}[\phi(p)-\phi(\mu)]$ is given by

$$
\operatorname{Pr}\left(b_{T} \leq s\right)=\Phi\left(\frac{s}{\sigma_{\phi}}\right)+O\left(T^{-1 / 2}\right)
$$

where $\sigma_{\phi}^{2}=d^{\prime} \Sigma d, d=\partial \phi(\mu) / \partial p$, and $\Phi($.$) is the standard normal c d f$. Sargan (1976) provided formulae for the coefficients of a refined approximation of the form

$\operatorname{Pr}\left(b_{T} \leq s\right)=\Phi\left(\frac{s}{\sigma_{\phi}}+\frac{h_{0}}{\sqrt{T}}+\frac{h_{1}}{T}\left(\frac{s}{\sigma_{\phi}}\right)+\frac{h_{2}}{\sqrt{T}}\left(\frac{s}{\sigma_{\phi}}\right)^{2}+\frac{h_{3}}{T}\left(\frac{s}{\sigma_{\phi}}\right)^{3}\right)+O\left(T^{-3 / 2}\right)$

where the coefficients $h_{j}$ were expressed as functions of the cumulants of $p$ and the derivatives of $\phi(p)$. In this way, Sargan showed an explicit method of finding Edgeworth approximations for a large class of statistics from linear-in-variables time series econometric models. [Successive corrections to these formulae appeared in Sargan's (1977) "Erratum", in Tse's (1981) Ph.D. thesis, and in Appendix B of Arellano and Sargan (1990); the first correct general formulae, however, were published using a slightly different notation in Phillips (1977).]

Resampling Methods Sargan (1976) was a remarkable paper in many other ways. The abundance of technical results was coupled with a genuine concern with the problems of applying the theoretical refinements to improving the accuracy of asymptotic significance tests in models of a realistic size. 
As an alternative to analytical Edgeworth expansions, Sargan (1976) suggested a resampling method (which he referred to as a "Barnard approximation" in implicit reference to Barnard's (1963) Monte Carlo testing). He argued that "an alternative approach, which has only rarely been used in econometrics, is to estimate the probability associated with a given significance test by simulating the model, using the estimated parameters, and observing the resulting proportion of simulated criteria falling within the asymptotic confidence interval". Sargan pointed out that at first sight the method suffered from depending upon the use of estimated parameters, but he noted that "the criteria have the property that their asymptotic distributions are independent of the parameters of the model. If we are considering a symmetric confidence interval for a $t$ ratio, the difference between the asymptotic probability and the finite sample probability is usually a differentiable function of the parameters in the neighborhood of the true value uniformly of order $1 / T$. It follows that the error in the estimated probability induced by using estimated values for these parameters is of order $T^{-3 / 2}$ ". He was, nevertheless, concerned that the asymptotic result might overstate the finite sample properties of the resampling method, and suggested to investigate the matter further through Monte Carlo experimentation.

These matters were pursued in Sargan (1981) [later briefly summarized in Sargan (1993)]. This paper studied the asymptotic properties of a parametric bootstrap procedure. It also undertook a Monte Carlo exercise using a twoequation overidentified dynamic model estimated by 3SLS to study the properties of his bootstrap method with $T=20$ and 50 . He found that the bootstrap procedure gave some very poor estimates of the true size of the various confidence intervals considered.

Approximations when the Number of Instruments is Large Sargan (1975b) argued that conventional asymptotic theory in which $T \rightarrow \infty$ and the model remains constant, was quite irrelevant for large models where the total number of variables was large relative to the sample size. As an alternative, he considered an asymptotic framework in which not only $T$ but also the number of instruments and the number of equations were tending to infinity. He focused on 
the estimation of a fixed number of parameters occurring in a subsystem of equations, and studied the asymptotic properties of an iterated instrumental variable estimator that used the over-identifying restrictions in forming the instruments (of the type considered by Brundy and Jorgenson 1971). Sargan's Theorem 3 established that the feasible and unfeasible IV estimators were asymptotically equivalent, and concluded that for these type of estimators the results of asymptotic theory were still a good approximation even in large models.

This paper pioneered a literature that focused on the properties of IV and GMM estimates when the number of moment conditions and the sample size tend to infinity (cf. Kunitomo 1980, Morimune 1983, and Bekker 1994).

\section{Serial Correlation}

The theory for the 1958 paper was developed under the assumption that the error $u_{t}$ was not autocorrelated. Sargan nevertheless regarded the assumption of lack of autocorrelation in measurement errors as being unrealistic, and pointed out that since his IV estimates were based on minimal assumptions they were still consistent if the errors were autocorrelated. Because of this he suggested that "it is probably not wise to use lagged values of a variable appearing in the relationship as instrumental variables" (1958, pp. 394 and 413). The idea was to rely on instruments that would not lose their validity in the event of serial correlation in measurement errors.

A contribution of Hansen's GMM perspective has been the following reaction to this problem: If the economic problem suggests that $E\left(g_{T}\right)=0$ but $\operatorname{Var}\left(g_{T}\right)$ differs from (3.2) because of serial dependence, let us obtain a consistent estimate $\widehat{\operatorname{Var}}\left(g_{T}\right)$ to perform optimal inference relative to the original moments $g_{T}$ [a similar perspective was present in the IV estimators proposed independently by Chamberlain (1982) and White (1982) for cross-sectional and panel data linear models with heteroskedasticity, and Cumby, Huizinga, and Obstfeld (1983) for linear rational expectations models]. To do this, one could specify a parametric process for $u_{t}$ or try to obtain a robust estimate of $\operatorname{Var}\left(g_{T}\right)$ under more general assumptions (as in Hansen and Hodrick 1980, and Hansen 1982). If the sample 
size is small the former may be a better idea than the latter, but even if the process for $u_{t}$ is misspecified the IV estimates will still be consistent. In any event, the suggested estimates will minimize

$$
g_{T}^{\prime}\left[\widehat{\operatorname{Var}}\left(g_{T}\right)\right]^{-1} g_{T}
$$

The motivation for Sargan's 1959 paper was the IV estimation of models with autocorrelated errors, but he did not follow the GMM route. Sargan specified a reduced form process for the errors and used this specification to change the orthogonality conditions. He then considered joint estimation of the structural parameters and those in the error process. By doing so he gained in efficiency but at the cost of a more fragile estimator (an early discussion of the pros and cons of these two approaches was in Griliches 1967, pp. 40-41).

Sargan considered an equation of the form

$$
a_{0}^{\prime} w_{t}^{\dagger}=\eta_{t}
$$

with autoregressive errors

$$
\eta_{t}=\sum_{j=1}^{J} \psi_{j} \eta_{t-j}+u_{t}
$$

leading to the transformed equation

$$
a_{0}^{\prime} w_{t}^{\dagger}-\sum_{j=1}^{J} \psi_{j} a_{0}^{\prime} w_{t-j}^{\dagger} \equiv \alpha\left(\theta_{0}\right)^{\prime} w_{t}=u_{t}
$$

where $\alpha\left(\theta_{0}\right)^{\prime}=\left(a_{0}^{\prime},-\psi_{1} a_{0}^{\prime}, \ldots,-\psi_{J} a_{0}^{\prime}\right)$ and

$$
w_{t}=\left(\begin{array}{l}
w_{t}^{\dagger} \\
w_{t-1}^{\dagger} \\
\vdots \\
w_{t-J}^{\dagger}
\end{array}\right) .
$$

He then suggested the joint estimation of the structural parameters $a_{0}$ and the autoregressive parameters $\psi_{j}$ from a set of non-linear moment conditions of the form:

$$
E\left(z_{t} u_{t}\right)=0
$$


[Specification tests of the common-factor bilinear restrictions in $\alpha\left(\theta_{0}\right)$ were studied in Sargan (1964) and Sargan (1980).]

To look at the nature of these moments in more detail, suppose that we have a vector $z_{t}^{\dagger}$ that provides valid instruments for the errors of the original equation, such that

$$
E\left(z_{t}^{\dagger} \eta_{t}\right)=0
$$

Sargan's 1958 estimator based on these moments would still be consistent even if the errors were serially correlated. If (4.6) holds and $\eta_{t}$ is serially correlated, in general we would also have $E\left(z_{t}^{\dagger} \eta_{t-j}\right)=0$ for $j=1, \ldots, J$ (e.g. if $z_{t}^{\dagger}$ were strictly exogenous instruments). If this is so then it will also be true that

$$
E\left(z_{t}^{\dagger} u_{t}\right) \equiv E\left(z_{t}^{\dagger} \eta_{t}\right)-\sum_{j=1}^{J} \psi_{j} E\left(z_{t}^{\dagger} \eta_{t-j}\right)=0 .
$$

However, the autoregressive parameters are not identified from the latter set of moments since they will hold not only for the true value of the $\psi_{j}$, but for any other value as well. Under the assumption of independence at all lags and leads between all latent variables, $u_{t}$ will also be orthogonal to $w_{t-1}^{\dagger}, \ldots, w_{t-J}^{\dagger}$, which can then be used as additional instruments to secure the identification of the $\psi_{j}$. The problem is that if the error process is misspecified, the IV estimates of the structural parameters jointly estimated with the $\psi_{j}$ will be inconsistent. On the other hand, if the error process is well specified, the joint estimates will be more efficient asymptotically than those based on $E\left(z_{t}^{\dagger} \eta_{t}\right)=0$ alone.

Sargan was aware that assuming a low order autoregressive process for $\eta_{t}$ was problematic conceptually, given that he was regarding the errors as a combination of structural shocks and measurement errors. He argued "it would appear logical to assume that each measurement error, and the random component, are being determined by a different mechanism, for example, by an autoregressive equation. However the problem of estimation which this assumption involves is very complicated, and indeed the only treatment which appears promising is that which assumes that the whole residual is determined by an autoregressive equation" (1959, p. 101). 
In later work, Sargan and co-authors considered structural dynamic models with serial correlation in various settings. Sargan (1961) studied the properties of ML estimates of dynamic simultaneous systems with VAR errors; Espasa and Sargan (1977) considered the spectral estimation of simultaneous systems with stationary errors, and Bhargava and Sargan (1983) analyzed models for short panels with unrestricted autocovariance matrix. In the last two papers completely exogenous instrumental variables were required to distinguish structural dynamics from the generic patterns of serial correlation employed.

\section{Nonlinear Instrumental Variable Estimation}

A remarkable contribution of Sargan's 1959 article was to develop in a general and rigorous way the instrumental variable estimation of nonlinear-in-parameters models. Sargan realized that the estimation of the transformed model (4.4) was a special case of a nonlinear in parameters model. So he set about to develop the theory for estimation of a general model in which parameter restrictions were expressed as functions of a smaller set of free parameters. In this way, the contribution of Sargan (1959) goes well beyond the resolution of the problem of estimating models with serially correlated errors that originally motivated the analysis.

The setting was

$$
\begin{aligned}
\alpha\left(\theta_{0}\right)^{\prime} w_{t} & =u_{t} \\
E\left(z_{t} u_{t}\right) & =0
\end{aligned}
$$

where $\alpha($.$) is a vector of q+1$ functions of a $k \times 1$ parameter vector $\theta$, and $z_{t}$ is an $r \times 1$ vector of instrumental variables.

I begin by considering the simple case where $r=k$. Sargan first considered the nature of the solutions of the set of $r$ equations:

$$
\bar{M}_{z w} \alpha\left(\theta_{0}\right) \equiv\left(p \lim _{T \rightarrow \infty} \frac{1}{T} \sum_{t=1}^{T} z_{t} w_{t}^{\prime}\right) \alpha\left(\theta_{0}\right)=0
$$

He did so because the behavior of the solutions of the sample moment equations

$$
\left(\frac{1}{T} \sum_{t=1}^{T} z_{t} w_{t}^{\prime}\right) \alpha(\theta)=0
$$


depends on the nature of the solutions to the limiting equations (5.3).

He distinguished three cases: (i) equations (5.3) have a unique solution, (ii) equations (5.3) have multiple solutions, and (iii) equations (5.3) have a continuous infinity of solutions so that they just determine a curve in $\theta$ space (the " $a$ priori unidentified case"). Moreover, in cases (i) and (ii) he also distinguished between singular and non-singular solutions. The value $\theta=\theta^{*}$ is a singular solution if $\bar{M}_{z w} \alpha\left(\theta^{*}\right)=0$ and the $r \times k$ Jacobian matrix

$$
\bar{M}_{z w} \frac{\partial \alpha\left(\theta^{*}\right)}{\partial \theta^{\prime}}
$$

does not have full rank.

Next, Sargan considered IV estimators that solve

$$
\min _{\theta} \alpha(\theta)^{\prime} M_{w z} M_{z z}^{-1} M_{z w} \alpha(\theta)
$$

and argued that the probability that there is a solution of (5.4) near each solution of (5.3) tends to unity as $T \rightarrow \infty$, provided that the solution is non-singular. Then he used a general consistency theorem for extremum estimators to establish consistency of the non-linear IV estimator. [This type of theorem, given without proof, predated a large literature in nonlinear econometrics. Its ideas were further elaborated in Sargan (1975c).]

The final step was to establish asymptotic normality. The result was

$$
\sqrt{T}\left(\widehat{\theta}-\theta_{0}\right) \stackrel{d}{\rightarrow} \mathcal{N}\left(0, \sigma^{2}\left[\left(\frac{\partial \alpha\left(\theta_{0}\right)}{\partial \theta^{\prime}}\right)^{\prime} \bar{M}_{w z} \bar{M}_{z z}^{-1} \bar{M}_{z w}\left(\frac{\partial \alpha\left(\theta_{0}\right)}{\partial \theta^{\prime}}\right)\right]^{-1}\right)
$$

where $\widehat{\theta}$ is the minimum of $\alpha(\theta)^{\prime} M_{w z} M_{z z}^{-1} M_{z w} \alpha(\theta)$ within or on the boundary of a small region in $\theta$ space surrounding a non-singular solution $\theta_{0}$ of the limiting equations (5.3).

Sargan then moved to the overidentified case where the number of instrumental variables is greater than the number of parameters $r>k$. As in the previous paper, he considered linear combinations of the $z_{t}$

$$
z_{t}^{*}=\Phi z_{t}
$$


where $\Phi$ is a $k \times r$ transformation matrix, showing that the optimal choice for $\Phi$ that minimizes the asymptotic variance is given by

$$
\Phi=\left(\frac{\partial \alpha\left(\theta_{0}\right)}{\partial \theta^{\prime}}\right)^{\prime} \bar{M}_{w z} \bar{M}_{z z}^{-1}
$$

so that the corresponding (unfeasible) estimates solve the equations

$$
\left(\frac{\partial \alpha\left(\theta_{0}\right)}{\partial \theta^{\prime}}\right)^{\prime} \bar{M}_{w z} \bar{M}_{z z}^{-1} M_{z w} \alpha(\theta)=0,
$$

and the asymptotic variance matrix is as in (5.6). Sargan (1959) argued that this suggests to consider the equations

$$
\left(\frac{\partial \alpha(\theta)}{\partial \theta^{\prime}}\right)^{\prime} M_{w z} M_{z z}^{-1} M_{z w} \alpha(\theta) \equiv \frac{\partial}{\partial \theta}\left[\alpha(\theta)^{\prime} M_{w z} M_{z z}^{-1} M_{z w} \alpha(\theta)\right]=0 .
$$

So he considered estimators that minimize the non-linear instrumental variable criterion $\alpha(\theta)^{\prime} M_{w z} M_{z z}^{-1} M_{z w} \alpha(\theta)$, establishing consistency and asymptotic normality with variance matrix equal to that in (5.6).

Next, Sargan briefly considered the singular case (which was further developed in his 1980 World Congress Presidential Address, Sargan 1983), where

$$
\operatorname{rank}\left(\bar{M}_{z w} \frac{\partial \alpha\left(\theta^{*}\right)}{\partial \theta^{\prime}}\right) \leq k-1
$$

He argued that in the case when the rank is $k-1$, "there is asymptotically a probability of $1 / 2$ that there is a single minimum with error of order $T^{-1 / 2}$ and a probability of $1 / 2$ of two minima with errors of order $T^{-1 / 4}$. However this case has a mainly academic interest since it is a priori unlikely that $\bar{M}_{z w}$ will take just those values which makes the solution singular, and if the solution is only almost singular the errors will be large but as in the previous sections."

The Minimax Approach Sargan (1959) generalized the minimax approach of his 1958 paper to the non-linear case. The idea is the same as before, namely, 
to minimize the largest squared correlation between the errors and a linear combination of the instruments:

$$
\min _{\theta}\left[\max _{\gamma} \frac{\left(\alpha(\theta)^{\prime} M_{w z} \gamma\right)^{2}}{\left(\alpha(\theta)^{\prime} M_{w w} \alpha(\theta)\right)\left(\gamma^{\prime} M_{z z} \gamma\right)}\right]=\min _{\theta} \lambda(\theta)
$$

where

$$
\lambda(\theta)=\frac{\alpha(\theta)^{\prime} M_{w z} M_{z z}^{-1} M_{z w} \alpha(\theta)}{\alpha(\theta)^{\prime} M_{w w} \alpha(\theta)} .
$$

Sargan showed that this estimator was asymptotically equivalent to the previous one, and argued that its advantage was that "in the application to the study of autoregressive residuals it gives a set of estimates symmetric between the different variables in the relationship", and also that the minimum $\hat{\lambda}_{1}$ provided the basis for "a significance test for the existence of a relationship of the proposed type". Next, he derived the asymptotic distribution of $T \widehat{\lambda}_{1}$ (the test statistic of overidentifying restrictions). The general theory concluded with discussions of testing for underidentification and of confidence regions.

\section{Concluding Remarks}

Sargan's 1958 and 1959 papers provided lasting foundations for the theory of instrumental variable estimation of linear and nonlinear-in-parameters models. His results and his way of thinking about econometric estimation are very much in the agendas of present-day econometricians and empirical economists.

Hansen (1982) extended Sargan's framework by considering fully non-linear models, but also by abstracting from equations and expressing an estimation problem as a list of moment conditions. Moreover, Sargan's departure from conventional asymptotic efficiency was taken a significant step further by Hansen. He considered orthogonality conditions defined in terms of errors prior to filtering to remove serial correlation, and generalized the properties of errors allowing for flexible forms of serial dependence and conditional heteroskedasticity.

Sargan's work on instrumental variables had also dispensed with likelihood functions and blended error in variables with simultaneity problems by relying on 
a given set of moment conditions. However, something was lost in the process of moving towards fully non-linear models, since in Sargan's original motivation measurement errors played an important role along side structural shocks. 


\section{References}

Amemiya, T. (1974): "The Nonlinear Two-Stage Least-Squares Estimator", Journal of Econometrics, 2, 105-110.

Anderson, T. W. and H. Rubin (1949): "Estimation of the Parameters of a Single Equation in a Complete System of Stochastic Equations", Annals of Mathematical Statistics, 20, 46-63.

Anderson, T. W. and H. Rubin (1950): "The asymptotic properties of Estimates of the Parameters of a Single Equation in a Complete System of Stochastic Equations", Annals of Mathematical Statistics, 20, 570-582.

Arellano, M. and J. D. Sargan (1990): "Imhof Approximations to Econometric Estimators", Review of Economic Studies, 57, 627-646.

Arellano, M., L. P. Hansen, and E. Sentana (1999): "Underidentification?", unpublished manuscript, CEMFI, Madrid.

Barnard, G. A. (1963): Contribution to Discussion on Professor Bartlett's Paper, Journal of the Royal Statistical Society, Series B, 25, 294.

Basmann, R. L. (1957): "A Generalized Classical Method of Linear Estimation of Coefficients in a Structural Equation", Econometrica, 25, 77-83.

Bekker, P.A. (1994): "Alternative Approximations to the Distributions of Instrumental Variable Estimators", Econometrica, 62, 657-681.

Bhargava, A. and J. D. Sargan (1983): "Estimating Dynamic Random Effects Models from Panel Data Covering Short Time Periods", Econometrica, 51, 1635-1659.

Brundy, J. M. and D. W. Jorgenson (1971): "Efficient Estimation of Simultaneous Equations by Instrumental Variables", Review of Economics and Statistics, 53, 207-224.

Chamberlain, G. (1982): "Multivariate Regression Models for Panel Data", Journal of Econometrics, 18, 5-46. 
Chernoff, H. and H. Rubin (1953): "Asymptotic Properties of Limited Information Estimates Under Generalized Conditions", Cowles Commission Monograph 14, 200-212.

Cumby, R. E., J. Huizinga, and M. Obstfeld (1983): "Two-Step Two-Stage Least Squares Estimation in Models with Rational Expectations", Journal of Econometrics, 21, 333-355; and (1983): "Errata", Journal of Econometrics, 23, 407.

Desai, M. J., D. F. Hendry, and G. E. Mizon (1997): "John Denis Sargan”, The Economic Journal, 107, 1121-1125.

Durbin, J. (1954): "Errors in Variables", Review of the International Statistical Institute, 22, 23-32.

Espasa, A. and J. D. Sargan (1977): "The Spectral Estimation of Simultaneous Equation Systems with Lagged Endogenous Variables", International Economic Review, 18, 583-605.

Geary, R. C. (1948): "Studies in Relations between Economic Time Series", Journal of the Royal Statistical Society. Series B, 10, 140-158.

Geary, R. C. (1949): "Determination of Linear Relations between Systematic Parts of Variables with Errors of Observation the Variances of Which Are Unknown", Econometrica, 17, 30-58.

Goldberger, A. S. (1972): "Structural Equation Methods in the Social Sciences", Econometrica, 40, 979-1001.

Griliches, Z. (1967): "Distributed Lags: A Survey", Econometrica, 35, 16-49.

Hansen, L.P. (1982): "Large Sample Properties of Generalized Method of Moments Estimators", Econometrica, 50, 1029-1054.

Hansen, L. P. and R. J. Hodrick (1980): "Forward Exchange Rates as Optimal Predictors of Future Spot Rates: An Econometric Analysis", Journal of Political Economy, 88, 829-853.

Hansen, L.P., J. Heaton, and A. Yaron (1996): "Finite Sample Properties of Some Alternative GMM Estimators", Journal of Business 83 Economic Statistics, 14, 262-280. 
Hausman, J. A. (1975): "An Instrumental Variable Approach to Full Information Estimators for Linear and Certain Nonlinear Econometric Models", Econometrica, 43, 727-738.

Heckman, J. J. (2000): "Causal Parameters and Policy Analysis in Economics: A Twentieth Century Retrospective", Quarterly Journal of Economics, 115, $45-97$.

Hendry, D. F. (1976): "The Structure of Simultaneous Equations Estimators", Journal of Econometrics, 4, 51-88.

Jorgenson, D. W. and J. Laffont (1974): "Efficient Estimation of Nonlinear Simultaneous Equations with Additive Disturbances", Annals of Economic and Social Measurement, 3, 615-640.

Kunitomo, N. (1980): "Asymptotic Expansions of the Distributions of Estimators in a Linear Functional Relationship and Simultaneous Equations", Journal of the American Statistical Society, 75, 693-700.

Maasoumi, E. (1988): "Denis Sargan and his Seminal Contributions to Economic and Econometric Theory". In Maasoumi, E. (ed.): Contributions to Econometrics: John Denis Sargan, Vol. 1, Cambridge University Press.

Morimune, K. (1983): "Approximate Distributions of $k$-Class Estimators when the Degree of Overidentifiability Is Large Compared with the Sample Size", Econometrica, 51, 821-841.

Phillips, P. C. B. (1977): "A General Theorem in the Theory of Asymptotic Expansions as Approximations to the Finite Sample Distributions of Econometric Estimators", Econometrica, 45, 1517-1534.

Phillips, P. C. B. (1985): "ET Interviews: Professor J. D. Sargan", Econometric Theory, 1, 119-139.

Reiers $\varnothing$, O. (1945): Confluence Analysis by Means of Instrumental Sets of Variables, Uppsala: Almquist and Wiksell.

Sargan, J. D. (1958): "The Estimation of Economic Relationships Using Instrumental Variables", Econometrica, 26, 393-415. 
Sargan, J. D. (1959): "The Estimation of Relationships with Autocorrelated Residuals by the Use of Instrumental Variables", Journal of the Royal Statistical Society. Series B, 21, 91-105.

Sargan, J. D. (1961): "The Maximum Likelihood Estimation of Economic Relationships with Autoregressive Residuals", Econometrica, 29, 414-426.

Sargan, J. D. (1964): "Wages and Prices in the United Kingdom: A Study in Econometric Methodology". In: P. E. Hart, G. Mills, and J. K. Whitaker (eds.): Econometric Analysis for National Economic Planning, London: Butterworths.

Sargan, J. D. (1975a): "Gram-Charlier Approximations Applied to $t$ Ratios of $k$-Class Estimators", Econometrica, 43, 327-346.

Sargan, J. D. (1975b): "Asymptotic Theory and Large Models", International Economic Review, 16, 75-91.

Sargan, J. D. (1975c): "The Identification and Estimation of Sets of Simultaneous Stochastic Equations", Unpublished discussion paper No. A1, LSE, November. Printed in: Maasoumi, E. (ed.): Contributions to Econometrics: John Denis Sargan, Vol. 1, Cambridge University Press, 1988.

Sargan, J. D. (1976): "Econometric Estimators and the Edgeworth Approximation", Econometrica, 44, 421-448; and (1977): "Erratum", Econometrica, 45,272 .

Sargan, J. D. (1980): "Some Tests of Dynamic Specification for a Single Equation", Econometrica, 48, 879-897.

Sargan, J. D. (1981): "Some Experiments with Post Estimation Simulation", LSE Econometrics Research Programme, unpublished manuscript.

Sargan, J. D. (1983): "Identification and Lack of Identification", Econometrica, $51,1605-1633$.

Sargan, J. D. (1993): "Some Alternatives to the Edgeworth Approximation for Econometric Statistics". In P. C. B. Phillips (ed.): Models, Methods, and Applications of Econometrics, Essays in Honor of A. R. Bergstrom, Blackwell. 
Sargan, J. D. and W. M. Mikhail (1971): "A General Approximation to the Distribution of Instrumental Variables Estimates", Econometrica, 39, 131169.

Stone, R. (1947): "On the Interdependence of Blocks of Transactions" (with discussion), Supplement to the Journal of the Royal Statistical Society, 9, $1-45$.

Theil, H. (1961): Economic Forecasts and Policy, Second edition, Amsterdam: North-Holland.

Tse, Y. K. (1981): "Edgeworth Approximation to the Finite Sample Distribution of Econometric Estimators and Test Statistics, unpublished Ph.D. thesis, University of London.

Wald, A. (1940): "The Fitting of Straight Lines if Both Variables are Subject to Error", Annals of Mathematical Statistics, 11, 284-300.

White, H. (1982): "Instrumental Variables Regression with Independent Observations", Econometrica, 50, 483-499.

Working, E. J. (1927): “What Do Statistical 'Demand Curves' Show?", Quarterly Journal of Economics, 41, 212-235.

Wright, P. G. (1928): The Tariff on Animal and Vegetable Oils, New York: Macmillan.

Wright, S. (1934): "The Method of Path Coefficients", Annals of Mathematical Statistics, 5, 161-215. 